ment which includes a cis-tran proline isomerization and an asparagine swapping the side-chain oxygen for a main-chain carbonyl. In contrast, several structures of other bound ligands shows the gate to the cavity in a closed conformation and one ligand disorders the gate so that it is no longer visible in the electron denisty. Solution-state kinetics studies indicate that this conformational change is not an artifact of the crystallization conditions. The trapping of the open conformation provides a unique view of the extent to which protein dynamics can allow small molecule penetration into "inaccesible" protein cavities.

Fitzgerald, M.M., Churchill, M.J., McRee, D.E., and Goodin, D.B. (1995). "Small Molecule Binding to an Artificially Created Cavity at the Active Site of Cytochrome c Peroxidase." Biochemistry 33, 3807-3818.

PS04.14.15 TOXIN INTO VACCINE: STUDIES OF AB5 BACTERIAL TOXINS. Ethan A Merrit, Ingeborg Feil, Win G J Hol: Dept of Biological Structure and HHMI, University of Washington, Seattle WA 98195-7742 Randall K Holmes: Dept of Microbiology, University of Colorado Health Sciences Center, Denver CO 80262, Rino Rappuoli: IRIS, Via Florentina 1, 53100 Siena, Italy

Cholera toxin and E. coli heat-labile enterotoxin are closely related $A B 5$ hexameric assemblies secreted into the intestine during bacterial infection. Together they are responsible for over a million deaths annually. As with many other bacterial toxins the catalytic activity resides in a separate ' $A$ ' subunit, while receptor binding and delivery of the toxin to the target cell is mediated by a separate ' $\mathrm{B}$ ' fragment, in this case a pentamer which binds to the oligosaccharide of ganglioside GM1.

In addition to their deleterious biological effect as toxins, however, these molecular assemblies exhibit a remarkable ability to stimulate the immune system. In particular they are capable of evoking a strong mucosal immune response when administered orally or intranasally, and have been reported to confer the same sort of evoked response to co-administered antigens. It is therefore of great interest to determine whether these toxins can be engineered to lose toxicity while retaining their immunological properties.

We report here our recent crystallographic studies aimed at understanding both carbohydrate recognition by the receptor binding site and substrate recognition by the catalytic site. In particular we report the structures of oligosaccharide complexes with mutant toxins exhibiting altered receptor binding specificity, and also the structure of an engineered mutant at the active site with altered toxicity.

PS04.14.16 THE STRUCTURE OF A DESIGNED PEPTIDE REFINED TO $2.1 \AA$ RESOLUTION. Nancy L. Ogihara, Manfred S. Weiss, William F. DeGrado, and David Eisenberg, UCLA-DOE Laboratory of Structural Biology and Molecular Medicine, Box 951570 , University of California, Los Angeles, Los Angeles, CA 90095-1570

The three-dimensional structure of the designed peptide Acetyl-E VEALEKK VAALESK VQALEKK VEALEHG-amide has been determined and refined to a crystallographic R-factor of $21.4 \%$ for all data from 10 to $2.1 \AA$, resolution. In the trigonal crystal, three molecules, related by a crystallographic 3 -fold axis form a parallel three helix bundle. The bundles are stacked headto- tail to form a continuous coiled coil along the $\mathrm{z}$ direction of the crystal. The contacts between neighboring helices within the coiled coiled are mainly hydrophobic; four layers of valine residues alternating with four layers of leucine residues form the core of the bundle. Mostly hydrophilic contacts mediate the interaction between trimers. Here, a total of 2 solvent mediated hydrogen bonds and 2 direct protein- protein hydrogen bonds are found. Based on the structure, we propose a rule for designing crystals of peptides containing continuous 2-, 3-, and 4-helix bundles.
PS04.14.17 BINDING OF SMALL ELECTRON-DENSE LIGANDS IN LARGE PROTEIN CAVITIES. Michael L. Quillin. Walter A. Baase, and Brian W. Matthews, Howard Hughes Medical Institute, Institute of Molecular Biology University of Oregon, Eugene, Oregon 97403.

The extent to which disordered water molecules occupy hydrophobic cavities in proteins has been the subject of considerable debate. In some cases, the techniques of NMR spectroscopy and $X$-ray crystallography have provided seemingly contradictory estimates of the solvent content of apolar cavities (Ernst et al. (1995), Science 267, 1813-1817; Matthews et al. (1995), Science 270,1847-1848). In an effort to resolve whether it is possible to detect fully occupied yet disordered molecules in a protein cavity using crystallographic methods, we have determined the crystal structures of several complexes of small, electron-dense molecules bound within cavity-containing mutants of T4 lysozyme. Two classes of probes have been studied in this manner: noble gases, such as krypton and xenon; and alkyl halides, including alkyl bromides and iodides. Although these ligands bind within the cavity, they do not do so in a disordered fashion. Rather, it appears that there are preferential binding sites which are highly conserved among the different ligands. Factors which influence the location of these sites will be discussed.

PS04.14.18 CRYSTAL STRUCTURE OFA STABLEALPHA1-ANTITRYPSIN VARIANT REVEALS THE STABLIZATION MECHANISM. SeongEon Ryu, Hee-Jung Choi, Kee Nyung Lee, Ki-Sun Kwon and Myeong-Hee Yu, Protein Engineering Division Korea Research Institute of Bioscience and Biotechnology, KIST P.O. Box 115, Yusong, Taejon 305-600, South Korea

$\alpha-1$-antitrypsin, which is a member of serpin family, controls the level of neutrophil elastase in plasma by inhibiting its activity. The conformation of the mobile reactive loop and the relative instability of the native $\alpha-1$-antitrypsin are implicated in the inhibitory mechanism. The crystal structure of a mutant form of the uncleaved $\alpha$-1-antitrypsin with stabilization mutations at seven different positions (hepta $\alpha$-1-antitrypsin) has been determined at $2.7 \AA$ resolution. The structure was compared with the structures of other serpins to understand the stabilizing effect of the mutations. We found that hepta $\alpha$-1-antitrypsin is stabilized by various mechanisms. i) relaxation of a conformational strain by removing unfavorable overlaps of Van der Waals radii. ii) stabilization of hydrophobic cores by addition of better hydrophobic interactions. iii) destabilization of the loop inserted structures. Among these, the stabilization by the relaxation of the conformational strain by the F51L mutation underscores the importance of the central hydrophobic core region in the sheet A opening of the serpins.

PS04.14.19 X-RAYANALYSIS OF HEAT-RESISTANT MUTANTS OF HU PROTEIN Takahiro Tominaga*, Shunsuke Kawamura, Makoto Kimura, Atsushi Nakagawa", Isao Tanaka", "Division of Biological Science, Graduate School of Science, Hokkaido University, Sapporo, 060, Japan, Laboratory of Biochemistry, Faculty of Agriculture, Kyushu University, Fukuoka, 812, Japan

HU protein is ubiquitous in eubacterial kingdom. This small basic protein of molecular weight 9,500 is also known as DNA bending protein. Its amino acid sequence is highly conserved, e.g., B.stearothermophilus and B.subtilis have only 12 different residues. However the HU proteins of the two species have quite different thermal denaturation temperature $\left(\right.$ B.st $65^{\circ} \mathrm{C}$, B.su $48^{\circ} \mathrm{C}$ ). To elucidate the relationship between structure and thermostability of B.st and B.suHUs, we constructed T13A, T33L, E34D, and $\mathrm{K} 38 \mathrm{~N}$ mutants where the amino acids in BstHU were changed to the corresponding ones in BsuHU. Mutant proteins were expressed, 
and purified. Tm values of each mutant were determined by circular dichroism $(C D)$ spectra. The change of $T m$ value $(\Delta T \mathrm{~m})$ from native BstHU was $+3.1^{\circ} \mathrm{C}(\mathrm{T} 13 \mathrm{~A}),+1.7^{\circ} \mathrm{C}(\mathrm{T} 33 \mathrm{~L}),-2.0^{\circ} \mathrm{C}(\mathrm{E} 34 \mathrm{D})$, and $-4.0^{\circ} \mathrm{C}(\mathrm{K} 38 \mathrm{~N})$ respectively. Each mutant was crystallized for $\mathrm{X}$-ray structure analysis. The crystallization conditions of each mutant were $40 \% \mathrm{MPD}, 80 \mathrm{mM}$ phosphate( $\mathrm{pH} 8.0$ ), and protein concentration was $25 \mathrm{mg} / \mathrm{ml}$. E34D mutant was crystallized as above condition plus $6 \%$ dioxane. $X$-ray experiments were done for T13A mutant, and this crystal diffracts at least $2.5 \AA$. Cell parameters are $a=65.5 b=37.3 c=65.5$ and $B=114.5$ (that is isomorphous to native B.st $\mathrm{HU}$ crystal), and overall merging-R was $3.8 \%$. Structure determination was done by a molecular replacement. For model structure, we used the coordinates of native B.st HU. Refinement of this mutant structure was done with $X$-plor and current $\mathrm{R}$-factor is $20 \%$. We discuss the difference of thermal stability between native and mutant based on the structure.

\section{Immune System}

MS04.15.01 STRUCTURAL BIOLOGY OF IgSF CELL ADHESION MOLECULES. E. Yvonne Jones. Laboratory of Molecular Biophysics, The Rex Richards Building, South Parks Rd., Oxford, OX1 3QU, UK.

Structural studies on cell adhesion molecules, primarily those belonging to the immunoglobulin superfamily (IgSF), will be described. Direct intercellular and cell-matrix interactions are fundamental to the development and maintenance of multicellular organisms. These interactions are mediated by the extracellular regions of specific cell surface receptors, termed cell adhesion molecules. Sequence analysis indicates that the extracellular regions of many of these molecules contain immunoglobulin-like folds, leading to their classification as members of the IgSF. IgSF molecules variously mediate specific adhesion through a broad range interaction modes, homotypic with the same or closely related IgSF molecules, heterotypic with other types of adhesion molecules or even with specific carbohydrates. We have determined the structures of functional portions of several IgSF adhesion molecules by $x$-ray crystallography. These include representative examples of molecules which function by widely differing adhesion modes; IgSF/IgSF, IgSF/integrin and IgSF/sialylated glycan. The structural adaptations of the basic Ig-like motif which confer the different binding specificities will be assessed.

Jones E.Y., Davis S.I. Williams A.F., Harlos K. and Stuart D.I. (1992) Crystal structure at $2.8 \AA$ resolution of a soluble form of the cell adhesion molecule CD2. Nature $360232-239$

Bodian D.L., Jones E.Y., Harlos K., Stuart D.I. and Davis S.J. (1994) Crystal structure of the extracellular region of the human cell adhesion molecule $\mathrm{CD} 2$ at $2.5 \AA$ resolution. Structure $2755-766$

Jones E.Y., Harlos K., Bottomley M.J., Robinson R.C., Driscoll P.C., Edwards R.M., Clements J.M., Dudgeon T.J. and Stuart D.I. (1995) Crystal structure of an integrin-binding fragment of Vascular cell adhesion molecule 1 (VCAM-1) at $1.8 \AA$ resolution.Nature $373539-544$

Vinson M., Van der Merwe P.A., Kelm S., May A., Jones E.Y. and Crocker P.R. (1996) Identification of a putative sialic acid binding site within the amino-terminal immunoglobulin-like domain of sialoadhesin: analysis by site-directed mutagenesis. J. Biol. Chem. (In the press)
MS04.15.02 THE CRYSTALSTRUCTURE OF ICAM-2, ITS COMPARISON TO OTHER INTEGRIN-BINDING PROTEINS. Wang, J-H.**, Casasnovas, J.*, Springer, T.*, Liu, J.H.*, Harrison, S.C.\$. "Center for Blood Research, Harvard Medical School, Boston, MA. 02115; fDept. of Mol. and Cell. Biol., Harvard University, Cambridge, MA. 02138; sDept. of Mol. and Cell. Biol. and HHMI, Harvard University, Cambridge, MA. 02138, USA

A crystal structure of the extracellular fragment of ICAM-2 has been determined to $2.2 \AA$ by the MIR method. ICAM- 2 is an adhesion molecule, constitutively expressed on endothelial cell surface; it binds to integrin LFA-1 on leukocyte surface. It may be important for leukocyte trafficking in uninflamed tissues. The structure consists of two tandomly arranged Ig domains; the compact, $N$-terminal integrin-binding domain (Di) and the relatively larger $C_{2}$-type Ig domain $\left(D_{2}\right)$. Both $D_{1}$ and $D_{2}$ are structurally homologous to $D_{1}-D_{2}$ of VCAM-1, with two distictions. The integrin-binding site on ICAM-2 is not so prominent a protrusion as in VCAM-1. Rather, significant bending between $D_{1}$ and $D_{2}$ ensures the exposure of the binding region. There are 5 "extra" residues at the $N$-terminus, flexibly extended from the tip of $D_{1}$. This extension may explain why ICAM-2 does not serve as a rhinovirus receptor despite the expected close structural similarity between ICAM-1 and ICAM-2.

MS04.15.03 STRUCTURAL STUDIES OF CATALYTIC ANTIBODIES WITH ESTERASE ACTIVITY. M. Knossow ${ }^{1}$, J.-B. Charbonnier1, B. Gigant 1 , B. Golinelli1, D. Tawfik 2 , Z. Eshhar ${ }^{2}$ and B.S. Green ${ }^{3}$ Laboratoire de Biologie Structurale, UMR 9920 C.N.R.S., 91198 Gif-sur-Yvette, France; 2Department of Chemical Immunology, Weizman Institute, Rehovot, Israël; 3 School of Pharmacy, Hebrew University of Jerusalem, Jerusalem, Israël.

The X-ray structures of the complexes of three esterase-like catalytic antibodies with a transition state analog of the reaction they catalyze are reported. These antibodies were identified by screening for catalytic activity the total immune repertoire elicited in response to the phosphonate transition state analog (TSA) hapten 1. The high resolution (1.9 $\AA$ and $2.2 \AA$ ) structures suggest that rate acceleration is accounted for by oxyanion stabilization.

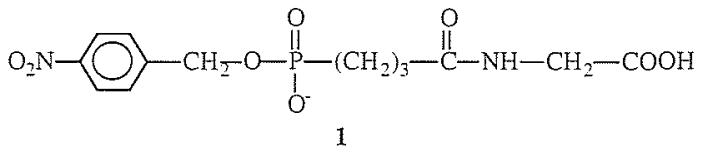

Most of the abzymes with esterase activities known to date have been raised against phosphonate haptens similar to 1 . Comparison of the newly determined structures to those of other abzymes with similar activities shows that the p-nitrophenol part of the hapten is buried in a hydrophobic pocket that is conserved in these antibodies and that the orientation of the hapten with respect to the antibody is conserved as well. The oxyanion hole motifs fund in Fabs with esterase-like activity whose structures have been determined are varied: a lysine (1), two peptide NHs (2) and an (Asn, Tyr) combination in the three antibodies we have studied. Participation to the oxyanion hole of a tyrosine residue is likely to be related to the well known prevalence of tyrosine residues in antibody combining sites; it is unprecedented in esterases.

(1)W.G. Zhou et al., Science 265, 1049-1064 (1994)

(2).J.-B. Charbonnier et al., P. N. A. S. USA 92, 11721-11725 (1995) 